Reprod. Nutr. Dévelop., 1983, 23 (4), 693-707.

\title{
Estimation in vivo de la composition corporelle de la brebis par la mesure de l'espace de diffusion de l'eau lourde
}

\author{
M. TISSIER, M. THÉRIEZ, A. PURROY $\left({ }^{*}\right)$, F. BOCQUIER \\ avec la collaboration technique de A. BRELURUT, J. LEROUX, J.-P. BRUN \\ Laboratoire de la Production ovine, I.N.R.A., \\ Theix, 63110 Beaumont, France, \\ (*) INIA, CRIDA 05, General Mola 41, Salamanca, Espagne.
}

Summary. In vivo estimate of ewe body composition by measuring the deuterium-oxide diffusion space.

Relationships for estimating body lipids, energy and proteins from the deuteriumoxide $\left(\mathrm{D}_{2} \mathrm{O}\right)$ diffusion space have been determined using 38 dry, pregnant or lactating ewes.

The animals were fed as usual and had free access to water up to slaughter. They were injected with deuterium-oxide in the morning, half an hour to 3 hours after being fed the whole daily ration or half of it. Blood samples were collected between 1 and $24 \mathrm{~h}$ after injection. The deuterium-oxide content of blood water was determined by infra-red spectrophotometry. The ewes were slaughtered $24 \mathrm{~h}$ after injection and their body water, protein, ash and energy were chemically determined.

Different blood samples were used to measure the deuterium-oxide diffusion space (theoretical initial concentration Co method). The closest relationship between measured body water $(E C M)$ and $D_{2} \mathrm{O}$ diffusion space $\left(E C D_{2} O\right)$ was found when the blood sample collected $8 \mathrm{~h}$ after injection was discarded.

Ewe body composition was estimated using a linear relationship in which ewe body weight and $\mathrm{D}_{2} \mathrm{O}$ diffusion space were two independent variable (relationship number 5 ). The best ewe body weight to use was the weight measured at injection time.

The estimation of body lipid was significantly affected by physiological status when slaughter weight and true body water, measured at slaughter, were used. The effect of this physiological status decreased when the water content of the digestive tract was taken into account and it was no longer significant when the water content of the uterus and conceptus was considered. Body composition could always be predicted by estimating the water content; the standard error was nearly $1.4 \mathrm{~kg}$. However, the method was not accurate enough to obtain a significant effect of physiological status on lipid estimation. Thus, at present we have proposed identical equations for dry, pregnant or lactating ewes.

\section{Introduction.}

Grâce à ses réserves corporelles, la brebis amortit les changements brusques du niveau des apports alimentaires ou de ses besoins. L'étude de régimes ali- 
mentaires et de leurs effets sur la production de l'animal ne peut donc être réalisée que si ces réserves corporelles et leurs variations sont connues.

Parmi les différentes méthodes disponibles pour obtenir ces résultats, l'estimation in vivo de la composition corporelle par mesure de l'espace de diffusion d'un marqueur est la plus satisfaisante (Robelin, 1973). Elle permet en particulier de décrire, sur le même animal, l'évolution des réserves énergétiques tout au long du cycle de reproduction et de les relier aux performances.

La majorité des travaux utilisant cette méthode ont été effectués sur des animaux en croissance ou sur des brebis vides (Panaretto, 1963, 1968 ; Searle, 1970a, b ; Farrell et Reardon, 1972 ; Donnelly et Freer, 1974 ; Robelin, 1977). Peu de travaux concernent les brebis gestantes (Foot et Greenhalgh, 1970). Par ailleurs, la plupart des auteurs, à l'exception de Foot et Greenhalgh (1970) et Robelin (1977), ont soumis les animaux à un jeûne solide et/ou hydrique allant jusqu'à $64 \mathrm{~h}$, lors de la mise au point de la méthode. Cette pratique, qui permet de réduire la variabilité du poids de contenu digestif, peut être appliquée sans inconvénient sur des adultes au repos.

Par contre, le jeûne peut perturber les femelles allaitantes et les femelles gestantes de races prolifiques dont il fait chuter la production laitière ou peut provoquer une toxémie de gestation.

Le but de cette mise au point était donc d'obtenir les relations d'estimation de la composition corporelle de la brebis à différents stades au cours de son cycle de reproduction à partir d'animaux nourris normalement pendant toute la durée de la mesure.

Nous avons utilisé l'eau lourde $\left(\mathrm{D}_{2} \mathrm{O}\right)$ comme marqueur car n'étant pas radioactive, elle peut être utilisée sans précautions particulières. De plus, nous en maîtrisons bien le dosage (Tissier et al., 1978).

\section{Matériel et méthodes.}

Animaux et conduite générale de l'expérimentation. - Les mesures ont porté sur 38 brebis adultes appartenant à 3 génotypes (lle-de-France : 2, Romanov $\times$ Limousines : 9 et Limousines : 27). Ces brebis ont été abattues à différents stades physiologiques: sèches (19 brebis), gestantes (7 brebis dont 2 au $100^{\mathrm{e}}$ jour de gestation, 4 au $125^{\mathrm{e}}$ jour et 1 au $140^{\mathrm{e}}$ jour) ou allaitantes (12 brabis dont 5 en première semaine de lactation et 7 en sixième semaine).

Les animaux étaient logés en case individuelle une semaine environ avant la date prévue pour l'abattage. Ils disposaient d'eau en permanence, de foin de pré et d'aliment concentré en quantités nécessaires pour satisfaire les apports alimentaires recommandés (Tissier et Thériez, 1978). L'eau et les aliments laissés à leur disposition pendant les périodes de mesures, étaient renouvelés tous les matins sauf le jour de l'abattage, celui-ci avait lieu vers $10 \mathrm{~h}$.

Deux séries d'abattages, correspondant à des procédures différentes, ont été effectuées :

- Série I : 20 brebis (17 sèches et 3 gestantes). Les animaux, qui recevaient la totalité de leur ration quotidienne en un seul apport le matin entre $7 \mathrm{~h} 30$ et $8 \mathrm{~h}$, 
ont été injectés la veille de l'abattage entre $9 \mathrm{~h} 30$ et $11 \mathrm{~h}$ (soit $2 \mathrm{~h} 30$ après le début de repas).

- Série II : 18 brebis (2 sèches, 4 gestantes et 12 allaitantes). Les rations quotidiennes étaient distribuées en 2 fois : entre $7 \mathrm{~h} 30$ et $8 \mathrm{~h}$ le matin, à $13 \mathrm{~h} 30$ l'après-midi. L'injection d'eau lourde a été réalisée entre $8 \mathrm{~h} 30$ et $10 \mathrm{~h}$, soit $1 \mathrm{~h}$ environ après le début du repas.

Les brebis ont été pesées à plusieurs reprises : à $14 \mathrm{~h}$ la veille de l'injection, puis le matin au moment de l'injection et $24 \mathrm{~h}$ plus tard avant abattage. Une pesée supplémentaire a été réalisée à $13 \mathrm{~h} 30$ le jour de l'injection (soit $7 \mathrm{~h}$ après celle-ci) sur les 18 brebis de la $2^{\mathrm{e}}$ série.

Injections d'eau lourde. - La technique utilisée pour l'injection a été décrite par Robelin (1977). La quantité d'eau lourde injectée était voisine de $0,6 \mathrm{~g} / \mathrm{kg}$ de poids vif ce qui correspond à des concentrations comprises entre 0,75 et 1,00 p. 1000 dans l'eau du sang.

Pour les brebis de la série $\mathrm{I}$, des prélèvements de sang ont été effectués 1 , $2,4,8,12$ et $24 \mathrm{~h}$ après l'injection pour déterminer la courbe d'élimination du marqueur. Dans la série II, les prélèvements de sang ont été faits $6,8,23,24 \mathrm{~h}$ après l'injection. Compte tenu des procédures utilisées, on notera que le prélèvement de $4 \mathrm{~h}$ de la série I et celui de $6 \mathrm{~h}$ de la série II ont été tous deux effectués entre 6 et $7 \mathrm{~h}$ après la distribution de la ration du matin.

Le sang prélevé dans la veine jugulaire grâce à des tubes de $10 \mathrm{ml}$, sous vide et héparinés, était conservé à $-20^{\circ} \mathrm{C}$ jusqu'à son traitement.

Abattage et mesures associées. - Après la dernière pesée (24 h après l'injection), les brebis, tondues, ont été abattues et saignées. Le sang a été recueilli en totalité et pesé. Les différents compartiments du tube digestif (rumen + réseau + feuillet, caillette, intestin grêle, gros intestin) ont été séparés et pesés pleins et vides. Les poids des contenus ont été obtenus par différence. Deux échantillons de sang et de contenus de chacun des compartiments du tube digestif ont été récoltés pour déterminer leur teneur en matière sèche.

Le reste du corps a été séparé en 3 parties :

- Les "viscères ": poumons + cœur + foie + rate + vessie, tube digestif vide, dépôts adipeux internes, utérus vide, mamelle ont été pesés séparément, puis rassemblés pour être broyés extemporanément ou après stockage à $-20^{\circ} \mathrm{C}$.

- La peau, la tête et les pattes (sabot, pied et os canon) ont été pesées, puis divisées en deux parties sensiblement équivalentes dont l'une a été conservée à $-20^{\circ} \mathrm{C}$ après pesée.

- La carcasse, pesée chaude puis stockée à $+4^{\circ} \mathrm{C}$ pendant $24 \mathrm{~h}$, a été pesée de nouveau puis fendue en deux parties équivalentes. On a séparé ensuite les muscles et le tissu adipeux du squelette de la moitié droite.

La mamelle, I'utérus et les fœus des brebis gestantes ont été traités séparément.

II a été procédé au broyage de chacune de ces parties, dans un broyeur à couteaux (viscères, peau, tête et pattes) après congélation dans l'azote liquide, dans un broyeur à vis (muscle + gras) ou avec une râpe (squelette). Dans cha- 
que cas, 3 échantillons ont été prélevés pour détermination de leur teneur en matière sèche et pour analyses.

Analyses chimiques. - Le traitement des échantillons de sang lextraction de l'eau, puis dosage de sa concentration en eau lourde) a été effectué suivant les méthodes décrites par Tissier et al. (1978).

La teneur en matière sèche des compartiments corporels a été déterminée sur 2 des 3 échantillons par passage à l'étuve à $80^{\circ} \mathrm{C}$ pendant $48 \mathrm{~h}$. Le troisième échantillon, lyophilisé, a servi à déterminer les teneurs en cendres (four à minéraliser), en protéines ( $N$ Kjeldahl $\times 6,25$ ) et en énergie (calorimétrie adiabatique). La quantité de lipides a été obtenue par différence. A titre de vérification, on a comparé l'énergie corporelle mesurée à celle que l'on peut calculer à partir des quantités de protéines et de lipides analysées. En retenant les valeurs calorifiques de 5,6 et $9,4 \mathrm{kcal} / \mathrm{g}$ respectivement pour les protéines et les lipides, l'énergie calculée est de $0,8 \pm 0,9 \%$ plus élevée que l'énergie mesurée, ce qui montre la bonne précision des analyses effectuées et la cohérence des résultats.

Définitions et notations. - A des fins de standardisation et pour simplifier l'exposé des résultats, nous proposons les définitions et notations suivantes :

- Eau corporelle mesurée, notée " ECM » : quantité d'eau corporelle mesurée par dessication après abattage des animaux.

- Eau corporelle du corps vide, notée " ECV 》 : eau corporelle mesurée, diminuée de l'eau des contenus digestifs.

- Espace de diffusion de l'eau lourde lou eau corporelle $\mathrm{D}_{2} \mathrm{O}$ ), notée " $\mathrm{ECD}_{2} \mathrm{O}$ " : volume ou quantité d'eau corporelle dans lequel le marqueur leau lourde) est supposé avoir diffusé de façon homogène.

- Eau corporelle estimée, notée "ECE »: quantité d'eau corporelle estimée grâce à l'équation de régression entre I'ECM et I'ECD $D_{2} \mathrm{O}$.

Bien que les brebis aient été tondues avant l'abattage et qu'on n'ait pas tenu compte de la laine dans la composition corporelle, les poids vifs utilisés dans les relations sont les poids des brebis en laine; ceci introduit une faible variabilité supplémentaire, mais permet d'appliquer les relations dans les conditions normales d'expérience, c'est-à-dire à des brebis en laine.

Dans la suite du texte, nous utiliserons trois poids différents qui sont : le poids à l'injection ( $P$ I dans la suite du texte ou dans les équations), le poids à l'abattage (PA) et le poids vif vide au moment de l'abattage (PVV). Les poids et les quantités d'eau sont toujours exprimés en $\mathrm{kg}$.

Méthodes de calcul de l'espace de diffusion de l'eau lourde. - Dans toute la suite des calculs destinés à déterminer les relations entre $E C M$ et $E C D_{2} \mathrm{O}$ ou à estimer les quantités de lipides, d'énergie et de protéines présentes dans l'animal, nous avons calculé l'espace de diffusion de l'eau lourde au moment de l'injection. Cet espace de diffusion à l'injection est égal au rapport entre la quantité d'eau lourde injectée $\left(Q_{0}\right)$ et la concentration initiale théorique de l'eau lourde dans l'organisme (Co) : $\mathrm{ECD}_{2} \mathrm{O}=\mathrm{Oo} / \mathrm{Co}$. La valeur Co est obtenue par extrapolation, au temps zéro, de la droite d'élimination du marqueur après équilibre (coordonnées semi-logarithmiques) (Robelin, 1973, 1977). 
Analyses statistiques. - L'étude statistique des données a été réalisée grâce à un programme d'analyse de variance-covariance. Les résultats sont décomposés selon le modèle suivant :

$$
Y_{i j}=a_{1}\left(x 1_{i j}\right)+a_{2}\left(x 2_{i j}\right)+a_{i}+a_{0}+e_{i j}
$$

$Y_{i j}$ est la variable à expliquer (quantité de lipides, protéines, énergie) de l'individu j et $x 1$ et $x 2$ sont deux covariables explicatrices, (poids et eau corporelle ou espace de diffusion). On peut aussi calculer un effet moyen du facteur a au niveau $\mathrm{i}$ : stade physiologique. Le terme $\mathrm{a}_{0}$ représente la valeur moyenne de $y$ commune à tous les individus et $\mathrm{e}_{\mathrm{ij}}$ le terme aléatoire propre à l'individu $\mathrm{j}$.

\section{Résultats.}

Nous avons reporté en annexe I la totalité des résultats individuels utilisés pour l'établissement des relations d'estimation et sur le tableau 1 les valeurs moyennes des différents groupes de brebis. Comme on peut le constater, si les poids vifs varient fortement à l'intérieur de chaque groupe de brebis (45 à $88 \mathrm{~kg}$ pour les brebis sèches par exemple), les variations des quantités de lipides sont encore beaucoup plus fortes (de 2,8 à $34,4 \mathrm{~kg}$ pour les brebis sèches). Cette forte variabilité a permis d'obtenir des relations applicables à la majeure partie des situations rencontrées dans la pratique.

\section{TABLEAU 1}

Caractéristiques et composition chimique des brebis abattues (moyennes et écarts types).

\begin{tabular}{|c|c|c|c|c|}
\hline État physiologique & sèches & gestantes & $\begin{array}{l}\text { allaitantes } \\
\text { (semaine }+1 \text { ) }\end{array}$ & $\begin{array}{l}\text { allaitantes } \\
\text { (semaine }+6 \text { ) }\end{array}$ \\
\hline Nombre & 19 & 7 & 5 & 7 \\
\hline $\begin{array}{l}\text { Poids vif à l'injection }(\mathrm{kg}) \\
\text { Poids vif à l'abattage (kg) }\end{array}$ & $\begin{array}{l}58,4(11,2) \\
57,2(10,3)\end{array}$ & $\begin{array}{l}68,9(11,2) \\
67,7(11,5)\end{array}$ & $\begin{array}{l}62,2(17,2) \\
61,4(16,4)\end{array}$ & 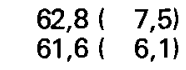 \\
\hline $\begin{array}{l}\text { Perte de poids (kg) entre } \\
\text { l'injection et l'abattage }\end{array}$ & $1,2(1,3)$ & $1,3(1,0)$ & $0,9(1,9)$ & $1,2(1,9)$ \\
\hline Poids vif vide $(\mathrm{PW})(\mathrm{kg})$ & $46,9(10,3)$ & $57,3(10,9)$ & $47,0(14,7)$ & $47,1(5,9)$ \\
\hline $\begin{array}{l}\text { Eau du corps entier (ECM) (kg) } \\
\text { Eau du contenu digestif }(\mathrm{kg})\end{array}$ & $\begin{aligned} 34,7 & (4,3) \\
9,0 & (2,2)\end{aligned}$ & $\begin{aligned} 41,9( & 7,7) \\
6,8 & (1,8)\end{aligned}$ & 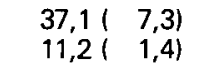 & $\begin{array}{ll}38,9 & (3,0) \\
12,0 & (2,5)\end{array}$ \\
\hline $\begin{array}{l}\text { Lipides (kg) } \\
\text { Protéines (kg) } \\
\text { Cendres (kg) } \\
\text { Energie (MJ) }\end{array}$ & 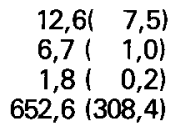 & $\begin{array}{r}13,8(\quad 2,5) \\
7,8(1,2) \\
2,0(0,2) \\
721,1(115,1)\end{array}$ & $\begin{array}{r}12,2(7,0) \\
7,0(\quad 1,6) \\
1,9(0,4) \\
643,3(307,5)\end{array}$ & $\begin{array}{r}11,3(4,4) \\
7,0(0,8) \\
2,0(0,1) \\
607,8(178,6)\end{array}$ \\
\hline $\begin{array}{l}\text { ECM \% PV abattage } \\
\text { Lipides \% PV abattage }\end{array}$ & $\begin{array}{l}60,7 \\
22,0\end{array}$ & $\begin{array}{l}61,9 \\
20,2\end{array}$ & $\begin{array}{l}60,4 \\
19,9\end{array}$ & $\begin{array}{l}63,1 \\
18,3\end{array}$ \\
\hline $\begin{array}{l}\text { Eau du corps vide \% PVV } \\
\text { Lipides \% PVV }\end{array}$ & $\begin{array}{l}54,8 \\
26,9\end{array}$ & $\begin{array}{l}59,0 \\
24,1\end{array}$ & $\begin{array}{l}55,1 \\
25,9\end{array}$ & $\begin{array}{l}57,0 \\
23,9\end{array}$ \\
\hline
\end{tabular}


Relations entre les différents composants du corps entier. - Nous avons soumis l'ensemble des données de composition chimique obtenues après abattage à une première analyse pour vérifier la validité des hypothèses servant de base à la méthode d'estimation.

Le poids vif à l'abattage explique à lui seul 70 à $82 \%$ de la variabilité des lipides, énergie et protéines. Comme pour Robelin (1977), ce sont les lipides qui restent les plus variables. L'introduction de I'ECM comme seconde variable indépendante améliore de façon hautement significative la précision des relations. L'écart type résiduel (ETR) des lipides passe, par exemple de près de $3,8 \mathrm{~kg}$ à moins de $0,8 \mathrm{~kg}$ lorsque l'ECM est incluse dans la relation avec le poids vif à l'abattage. L'amélioration est moindre pour les protéines car le poids seul explique déjà $82 \%$ de la variabilité.

La relation calculée pour les lipides (L) sur l'ensemble des brebis est la suivante :

(1) $L_{k g}=0,940 \mathrm{PA}_{\mathrm{kg}}-1,149 \mathrm{ECM}_{\mathrm{kg}}-1,656 ; \mathrm{R}^{2}=0,984$ et $\mathrm{ETR}_{\mathrm{kg}}=0,970$.

Cette relation a été également calculée en exprimant les quantités d'eau et de lipides corporels en pourcentage du poids à l'abattage (fig. 1, points noirs) : (2) $\quad \mathrm{L} \% \mathrm{PA}=93,770-1,192 \mathrm{ECM} \% \mathrm{PA} ; \mathrm{R}^{2}=0,963$ et $\mathrm{ETR} \%=1,38$.

Relation entre l'eau corporelle mesurée (ECM) et l'espace de diffusion de I'eau lourde $\left(\mathrm{ECD}_{2} \mathrm{O}\right)$. - Les différentes possibilités de calcul de $\mathrm{ECD}_{2} \mathrm{O}$ à partir de 2 à 4 prélèvements de sang sont présentées dans le tableau 2 . Les équations établies ont un coefficient de corrélation très élevé $\left(R^{2}\right.$ est toujours supérieur

\section{TABLEAU 2}

Relation entre l'eau corpore/le mesurée et l'espace de diffusion de l'eau lourde $\left(\mathrm{ECD}_{2} \mathrm{O}\right)$. Effets de la méthode de détermination de $\mathrm{ECD}_{2} \mathrm{O}$ et de la variation de poids entre l'injection et l'abattage sur la précision de cette relation.

\begin{tabular}{|c|c|c|c|c|c|c|}
\hline & & \multirow{3}{*}{$\begin{array}{l}\text { Nombre et heures des } \\
\text { prélèvements utilisés }\end{array}$} & \multicolumn{4}{|c|}{ Variables indépendantes } \\
\hline & & & \multicolumn{2}{|c|}{$\mathrm{ECD}_{2} \mathrm{O}$} & \multicolumn{2}{|c|}{$\begin{array}{l}\mathrm{ECD}_{2} \mathrm{O} \text { et variation de poids } \\
\text { entre l'injection et l'abattage }\end{array}$} \\
\hline & & & $\mathbf{R}^{2}$ & ETR $(k g)$ & $R^{2}(1)$ & $\operatorname{ETR}(\mathrm{kg})$ \\
\hline série I & $\begin{array}{l}4 \\
3 \\
3 \\
2 \\
2\end{array}$ & 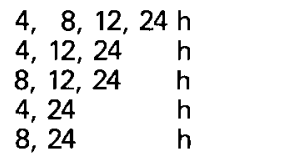 & $\begin{array}{l}0,939 \\
0,933 \\
0,943 \\
0,924 \\
0,940\end{array}$ & $\begin{array}{l}1,079 \\
1,134 \\
1,046 \\
1,202 \\
1,072\end{array}$ & $\begin{array}{l}0,962^{* *} \\
0,962^{* *} \\
0,953^{*} \\
0,959^{* *} \\
0,949 \text { NS }\end{array}$ & $\begin{array}{l}0,881 \\
0,875 \\
0,980 \\
0,914 \\
1,017\end{array}$ \\
\hline série II & $\begin{array}{l}4 \\
3 \\
3 \\
2 \\
2\end{array}$ & $\begin{array}{lr}6,8,23,24 \mathrm{~h} \\
6,23,24 & \mathrm{~h} \\
8,23,24 & \mathrm{~h} \\
6,24 & \mathrm{~h} \\
8,24 & \mathrm{~h}\end{array}$ & $\begin{array}{l}0,924 \\
0,930 \\
0,895 \\
0,929 \\
0,892\end{array}$ & $\begin{array}{l}1,911 \\
1,836 \\
2,250 \\
1,852 \\
2,280\end{array}$ & $\begin{array}{l}0,952^{* *} \\
0,960^{* *} \\
0,917^{*} \\
0,959^{* *} \\
0,915^{*}\end{array}$ & $\begin{array}{l}1,561 \\
1,433 \\
2,063 \\
1,443 \\
2,088\end{array}$ \\
\hline
\end{tabular}

(1) NS, ${ }^{*},{ }^{*}$ : la seconde variable (variation de poids) améliore de manière non significative (NS) ou de manière significative au seuil de $5 \%\left(^{*}\right)$ ou $1 \%\left(^{* *}\right)$ la relation obtenue avec la première variable $\left(\mathrm{ECD}_{2} \mathrm{O}\right)$. 


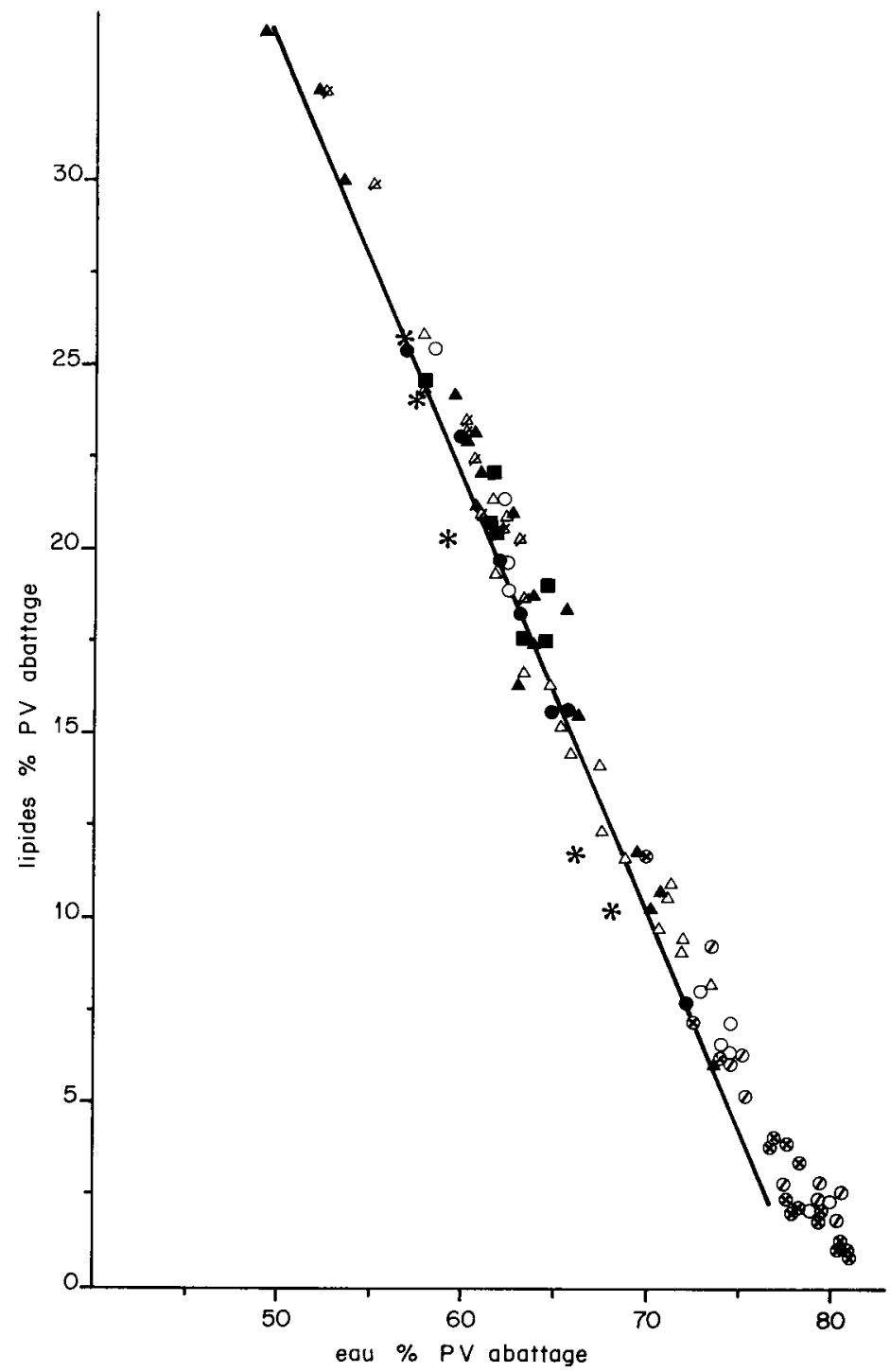

FIG. 1. - Relation entre les quantités de lipides et d'eau corporelle exprimées en pourcentage du poids vif à l'abattage.

1 - points blancs, données de Foot, Skedd et Mc Farlane (1979) : $\Delta$ et $\Delta$ Blackface sèches ; $\bigcirc$ Blackface allaitantes; $\varnothing$ Greyface milieu de lactation; $\otimes$ Greyface sevrage.

2 - points noirs, données présentes; $\boldsymbol{\Delta}$ sèches ; $\mathbf{q}$ gestantes; ${ }^{*}$ allaitantes semaine +1 ; - allaitantes semaine +6 . 
à 0,89 ). L'écart type résiduel varie de 1 à $2,3 \mathrm{~kg}$ selon le nombre de prélèvements utilisés et les intervalles de temps entre l'injection et les prises de sang; il est plus faible pour la série I que pour la série II.

Ces relations sont améliorées de manière le plus souvent hautement significative lorsque la variation de poids entre l'injection et l'abattage $(\Delta P=-1,8 \mathrm{~kg}$ en moyenne) est utilisée comme seconde variable indépendante (tabl. 2), par contre, l'amélioration est faible ( $p<0.05$ ou NS) lorsque les prélèvements $8 \mathrm{~h}$ après l'injection sont inclus dans la droite de régression qui permet de calculer Co.

Par conséquent, les meilleures relations entre $\mathrm{ECM}$ d'une part, $E C \mathrm{D}_{2} \mathrm{O}$ et $\triangle \mathrm{P}$ $\mathrm{d}^{\prime}$ autre part, sont celles qui ont été établies sans la prise de sang $8 \mathrm{~h}$ après l'injection, donc avec 2 ou 3 prises de sang pour le calcul de Co.

Ces relations permettent inversement de calculer l'eau corporelle d'une brebis dont on connait l'espace de diffusion $\left(\mathrm{ECD}_{2} \mathrm{O}\right)$ et, éventuellement, la variation de poids entre l'injection et l'abattage lou la dernière prise de sang qui correspond à l'abattage).

Pour l'ensemble des 38 brebis analysées, l'eau corporelle ECM est égale à :

(3) $\mathrm{ECM}_{\mathrm{kg}}=4,424+0,829 \mathrm{ECD}_{2} \mathrm{O}_{\mathrm{kg}}$;

$$
\mathrm{R}^{2}=0,939 \text { et } \mathrm{ETR}_{\mathrm{kg}}=1,468
$$

(4) $\mathrm{ECM}_{\mathrm{kg}}=3,997+0,863 \mathrm{ECD}_{2} \mathrm{O}_{\mathrm{kg}}-0,636 \Delta \mathrm{P}_{\mathrm{kg}}$

$$
R^{2}=0,955 \text { et } \mathrm{ETR}_{\mathrm{kg}}=1,274
$$

$\mathrm{ECD}_{2} \mathrm{O}$ étant déterminé à partir de 3 prises de sang $(4,6$ et $24 \mathrm{~h}$ après l'injection).

Relation d'estimation des lipides à partir de $\mathrm{ECD}_{2} \mathrm{O}$ et du poids vif. - Différentes relations d'estimation des lipides à partir de l'espace de diffusion et du poids vif ont été établies selon le nombre de prises de sang utilisées et le poids vif déterminé à différents moments (injection, abattage, poids moyen au cours des $24 \mathrm{~h}$ de mesure) pour chacune des deux séries d'abattage.

Comme dans le cas précédent (relation entre eau corporelle et espace de diffusion) ce sont les estimations de l'espace de diffusion obtenues à partir de 2 ou 3 prises de sang qui sont les plus précises, en éliminant la prise de sang de $8 \mathrm{~h}$.

Le poids correspondant à la meilleure précision est le poids à l'injection lorsqu'il est associé à $\mathrm{ECD}_{2} \mathrm{O}$ (tabl. 3) alors que c'est le poids d'abattage qui donne la meilleure relation lorsqu'on dispose de l'ECM.

On a également calculé le poids moyen des animaux à partir de 3 ou 4 pesées : injection, abattage (pesées du matin, c'est-à-dire peu après la distribution des rations) et 1 ou 2 pesées de l'après-midi lorsqu'une grande partie des rations a été consommée. L'utilisation de ce poids moyen dans la relation d'estimation des lipides se traduit par un écart type résiduel très voisin de celui obtenu avec le poids à l'injection.

La quantité de lipides (L) peut donc être calculée à partir du poids à l'injection (PI) et de l'espace de diffusion $\left(\mathrm{ECD}_{2} \mathrm{O}\right)$ estimé à partir de 3 prises de sang réalisées 4,6 et $24 \mathrm{~h}$ après l'injection à l'aide de la relation (5) :

(5) $\mathrm{L}_{\mathrm{kg}}=0,861 \mathrm{Pi}_{\mathrm{kg}}-0,911 \mathrm{ECD}_{2} \mathrm{O}_{\mathrm{kg}}-4,654 ; \mathrm{R}^{2}=0,965$ et $\mathrm{ETR}_{\mathrm{kg}}=1,78$. 
TABLEAU 3

Relations d'estimation des lipides à partir du poids vif (injection ou abattage) et de l'eau corporelle mesurée (ECM) ou de l'espace de diffusion ( $\left.\mathrm{ECD}_{2} \mathrm{O}\right)$.

Influence des variations de poids entre injection et abattage.

\begin{tabular}{|c|c|c|c|c|c|c|c|}
\hline \multicolumn{5}{|c|}{ Coefficients des variables indépendantes } & \multirow{2}{*}{$\begin{array}{l}\text { Terme } \\
\text { constant }\end{array}$} & \multirow[b]{2}{*}{$\mathbf{R}^{2}$} & \multirow[b]{2}{*}{ ETR $(\mathrm{kg})$} \\
\hline \multicolumn{2}{|c|}{$\begin{array}{l}\text { Poids vif } \\
\text { à l'abattage à l'injection }\end{array}$} & \multicolumn{2}{|c|}{$\begin{array}{l}\text { Eau corporelle } \\
\mathrm{ECM} \quad \mathrm{ECD}_{2} \mathrm{O}\end{array}$} & $\begin{array}{c}\text { Variation de } \\
\text { poids }\end{array}$ & & & \\
\hline 0,940 & - & $-1,149$ & - & - & $-1,656$ & 0,984 & 0,790 \\
\hline- & 0,826 & $-1,003$ & - & - & $-1,199$ & 0,956 & 1,313 \\
\hline 0,904 & - & - & $-0,916$ & - & $-6,058$ & 0,934 & 1,610 \\
\hline - & 0,861 & - & $-0,911$ & - & $-4,654$ & 0,965 & 1,178 \\
\hline 0,864 & - & - & $-0,913$ & $-0,838$ & $-4,700$ & 0,955 & 1,188 \\
\hline
\end{tabular}

Les effets des variables indépendantes présentées ci-dessus sont toujours hautement significatifs $(P<0,01)$.

Estimation de l'énergie et des protéines corporelles. - Des relations analogues à celles établies pour l'eau et les lipides ont été calculées pour les protéines et l'énergie corporelles.

Les résultats correspondants figurent dans le tableau 4 dans lequel nous avons reporté deux types de relations : celles qui relient les protéines et/ou I'énergie corporelles à $\mathrm{ECM}$ et au poids d'abattage PA d'une part, à $\mathrm{ECD}_{2} \mathrm{O}$ et au poids à l'injection $\mathrm{PI}$ d'autre part.

Effet du stade physiologique des brebis sur leur composition corporelle et sur les relations d'estimation. - Les 38 brebis abattues se trouvaient à 4 stades physiologiques différents : taries, fin de gestation, $1^{\text {re }}$ ou $6^{\mathrm{e}}$ semaine de lactation. Bien que les effectifs de ces 4 groupes soient très différents $119,7,5$ et 7 animaux respectivement pour les 4 stades) nous avons calculé l'ensemble des relations précédentes une seconde fois, soit en introduisant le stade physiologi-

\section{TABLEAU 4}

Relations entre les protéines ou l'énergie corporelle, le poids vif (injection ou abattage) et l'eau corporelle mesurée (ECM) ou l'espace de diffusion de l'eau lourde $\left(\mathrm{ECD}_{2} \mathrm{O}\right)$.

\begin{tabular}{|c|c|c|c|c|c|c|c|}
\hline & \multicolumn{4}{|c|}{ Coefficient des variables indépendantes } & \multirow{2}{*}{$\begin{array}{c}\text { Terme } \\
\text { constant }\end{array}$} & \multirow[b]{2}{*}{$R^{2}$} & \multirow[b]{2}{*}{ ETR } \\
\hline & $\begin{array}{l}\text { Poic } \\
\text { à l'abattage }\end{array}$ & $\begin{array}{l}\text { Is vif } \\
\text { à l'injection }\end{array}$ & $\begin{array}{l}\text { Eau co } \\
\text { ECM }\end{array}$ & $\mathrm{ECD}_{2} \mathrm{O}$ & & & \\
\hline $\begin{array}{l}\text { Protéines } \\
\text { corporelles } \\
(\mathrm{kg})\end{array}$ & $\begin{array}{c}0,054 \\
-\end{array}$ & $0, \overline{048}$ & $+\underset{-}{0,079}$ & $+\overline{0,076}$ & $\begin{array}{r}+\quad 0,793 \\
+\quad 1,055\end{array}$ & $\begin{array}{l}0,859 \\
0,882\end{array}$ & $\begin{array}{l}0,427 \\
0,391\end{array}$ \\
\hline $\begin{array}{l}\text { Energie } \\
\text { corporelle } \\
\text { (Mcal) }\end{array}$ & $\begin{array}{c}9,137 \\
-\end{array}$ & $8, \overline{378}$ & $\begin{array}{c}10,349 \\
-\end{array}$ & $-\overline{8}, 153$ & $\begin{array}{l}-12,725 \\
-39,160\end{array}$ & $\begin{array}{l}0,985 \\
0,971\end{array}$ & $\begin{array}{r}7,782 \\
10,748\end{array}$ \\
\hline
\end{tabular}

Les effets des variables indépendantes sont toujours hautement significatifs ( $P<0,001$ ). 
que comme facteur dans l'analyse de covariance, soit en traitant séparément chacun des lots et en testant la signification des relations obtenues.

La relation (6) entre les lipides (L), le poids d'abattage (PA) et I'eau corporelle (ECM) établie en tenant compte du stade physiologique (S) est la suivante : (6) $L_{k g}=0,937 P_{k g}-1,125 E C M_{k g}+S_{k g} ; R^{2}=0,992$ et $E T R_{k g}=0,580$ avec $S=-1,990 \mathrm{~kg}$ pour les brebis sèches, $-2,534$ pour les brebis gestantes, - 3,631 pour les brebis en $1^{\text {re }}$ semaine de lactation et - 2,791 pour les brebis en $6^{\mathbf{e}}$ semaine de lactation. Les valeurs de $\mathrm{S}$ étant significativement différentes au seuil de $P<0,001$.

Cette relation (6) est plus précise que l'équation (1) établie pour l'ensemble des animaux sans tenir compte du stade physiologique : $\mathrm{R}^{2}=0,992$ vs 0,984 et l'écart type résiduel (ETR) est réduit de 0,790 à $0,580 \mathrm{~kg}$.

Un calcul analogue réalisé pour l'énergie corporelle montre que la prise en compte du stade physiologique améliore de manière hautement significative la précision de la relation entre l'énergie corporelle, le poids à l'abattage et l'eau corporelle mesurée. Par contre, le facteur stade physiologique n'a aucun effet significatif sur la précision de la relation correspondant aux protéines corporelles.

Lorsque $\mathrm{PI}$ et $E C D_{2} \mathrm{O}$ sont utilisés, à la place de $\mathrm{PA}$ et ECM, dans les équations d'estimation des lipides, de l'énergie et des protéines corporelles, l'effet stade physiologique n'est jamais significatif. Ceci ne nous permet donc pas de proposer des équations d'estimation différentes selon le stade physiologique.

\section{Discussion.}

La relation d'estimation des lipides à partir du poids à l'abattage (PA) et de l'ECM est d'une bonne précision ; l'écart type résiduel est de $0,58 \mathrm{~kg}$, soit $4,5 \%$ du poids moyen des lipides estimés en tenant compte des différences liées au stade physiologique. On a comparé ces résultats à ceux disponibles dans la bibliographie et rassemblé dans ce but 114 autres données individuelles sur des ovins adultes, mâles castrés ou brebis à différents stades physiologiques. Les relations calculées successivement sur 114 données, puis sur 152 données, en incluant les nôtres, sont les suivantes :

$$
\begin{array}{rl}
\text { (7) } L_{k g}=0,922 P_{k g}-1,129 \mathrm{ECM}_{\mathrm{kg}}+1,739 & \mathrm{n}=114 ; \\
& \mathrm{R}^{2}=0,982 \text { et } \mathrm{ETR}_{\mathrm{kg}}=0,974 \\
\text { (8) } \mathrm{L}_{\mathrm{kg}}=0,931 \mathrm{PA}_{\mathrm{kg}}-1,136 \mathrm{ECM}_{\mathrm{kg}}-1,850 & \mathrm{n}=152 ; \\
\mathrm{R}^{2}=0,984 \text { et } \mathrm{ETR}_{\mathrm{kg}}=0,938 .
\end{array}
$$

L'introduction de nos 38 données ne modifie pas significativement l'équation globale (7) qui est tout à fait comparable à celle que nous avions établie sur nos seules données (équation (1)).

La précision de ces équations est tout à fait satisfaisante compte tenu de la diversité des animaux utilisés et des méthodes d'analyses correspondant à 10 laboratoires différents.

Leur écart type résiduel peut être réduit en tenant compte d'un effet auteur (hautement significatif) qui regroupe les différences dues au type d'animal (mâles castrés ou brebis), à son stade physiologique, à son état d'engraissement, ainsi qu'aux techniques de mesures à l'abattage et aux analyses. De plus, ces rela- 
tions ne sont pas linéaires et leur écart type résiduel est encore réduit si on introduit un terme quadratique $(\mathrm{PA})^{2}$ ou $(\mathrm{ECM})^{2}$. Cette non-linéarité est bien démontrée par les seuls résultats de Foot, Skedd et Mc Farlane (1979) (fig. 1) avec des brebis très maigres.

Ces calculs montrent donc la portée générale des relations entre les composants corporels déjà bien établies sur les animaux en croissance (cf. Robelin, $1973,1977)$ et justifient leur utilisation à des fins de prédiction de la composition corporelle des brebis adultes. On comprend alors que la précision de cette prédiction dépend en grande partie de la précision avec laquelle on peut estimer l'eau corporelle grâce à l'eau lourde.

Estimation de l'eau corporelle grâce à l'eau lourde. - L'étude faite pour les relations d'estimation de l'ECM à partir de $\mathrm{I}^{\prime} E \mathrm{CD}_{2} \mathrm{O}$ et des variations de poids permet de discuter plusieurs points importants : le moment de l'équilibre, la façon de calculer l' $E C D_{2} \mathrm{O}$ en relation avec l'effet de la distribution de la ration et le meilleur poids à associer à $\mathrm{I}^{\prime} \mathrm{EC} \mathrm{D}_{2} \mathrm{O}$.

Les dosages d'eau lourde réalisés au cours de la première série de mesures montrent que l'équilibre après diffusion complète de l'eau lourde semble être atteint tôt après l'injection et en tout cas $4 \mathrm{~h}$ après cette dernière dans la série $\mathrm{I}$ d'abattages constitués essentiellement de brebis vides. Pour la série II, avec une majorité de brebis allaitantes, l'équilibre serait atteint au maximum $6 \mathrm{~h}$ après l'injection et peut être même avant, sans que l'on dispose toutefois des prélèvements pour le prouver; en effet, pour ces brebis, la vitesse de renouvellement de l'eau est élevée mais, à l'inverse, les contenus digestifs plus importants peuvent augmenter la durée de diffusion. D'autres auteurs ont trouvé des durées de diffusion comparables aux nôtres : $4 \mathrm{~h}$ pour Searle (1970) sur des brebis ou mâles castrés de plus de 4 semaines d'âge et $5 \mathrm{~h}$ pour Till et Downes (1962) pour des ovins adultes. Par contre, Smith et Sykes (1974) trouvent que l'équilibre est tout juste atteint $8 \mathrm{~h}$ après l'injection. Tous ces auteurs ont pourtant utilisé le même marqueur, l'eau tritiée, mais avec des périodes de jeûne de 16 à $24 \mathrm{~h}$ avant l'injection et de 0 à $8 \mathrm{~h}$ après cette dernière, ce qui peut expliquer une partie des différences: Smith et Sykes (1974) pensent que la suppression des aliments et de l'eau peut accroitre le temps de diffusion du fait de la diminution de la sécrétion salivaire. De la même manière, Cowan et al. (1979) dont les brebis étaient privées d'eau entre l'injection et l'abattage, ont obtenu des temps d'équilibre différents chez les brebis au 12,41 et $111^{\mathrm{e}}$ jour de lactation, ce qui a modifié la relation entre $E C M$ et $E C D_{2} \mathrm{O}$ et a nécessité l'emploi de relations d'estimation différentes selon le stade.

Dans notre cas, l'alimentation normale des brebis a sans doute favorisé la diffusion rapide du marqueur que l'on a observée. Elle pose cependant d'autres problèmes. L'existence d'un rythme nycthéméral d'ingestion d'aliments et d'eau, suite à la (ou aux) distribution(s) de la ration, rend variable l'élimination du marqueur au cours des $24 \mathrm{~h}$ qui suivent l'injection en entrainnant une dilution artificiellement élevée de l'eau lourde dans l'eau corporelle. Les baisses rapides de concentration entre les prélèvements 4 (ou 6) et 8 h après l'injection en sont la preuve. Ceci explique vraisemblablement pourquoi l'utilisation de ce prélèvement

Reproduction Nutrition Développement, $n^{\circ} 4-1983$. -5 . 
$8 \mathrm{~h}$ après l'injection pour calculer Co aboutit non seulement à une surestimation plus importante de l'ECM par l'ECD $\mathrm{I}_{2} \mathrm{O}$, mais aussi à une plus forte variabilité résiduelle de la relation entre ces deux variables. Ces perturbations liées à l'ingestion d'aliments et d'eau sont probablement en partie responsables des moins bons résultats obtenus sur les brebis de la série II d'abattage par rapport à la série I. Pour préciser ces résultats, une étude spécifique plus approfondie reste nécessaire, elle pourra également apporter des données supplémentaires pour les brebis gestantes et allaitantes.

Pour l'application de la méthode, on peut donc retenir une procédure intermédiaire entre celles utilisées pour les séries I et II, c'est-à-dire une première distribution de la ration le matin $2 \mathrm{~h}$ avant l'injection, 1 ou 2 prélèvements de sang 5 à $6 \mathrm{~h}$ après l'injection suivi(s) éventuellement d'une deuxième distribution de la ration et, le lendemain, d'un autre prélèvement de sang dans le même intervalle par rapport aux repas. Cette méthode de détermination de l'espace de diffusion nous permet de nous affranchir de la difficulté d'estimer le temps d'équilibre. Ceci est confirmé, dans notre cas par le fait que la relation entre ECM et $E D_{2} \mathrm{O}$ (relation 3) est indépendante du stade physiologique contrairement à Cowan et al. (1979) qui utilisent la méthode du prélèvement à l'équilibre.

En ce qui concerne le poids vif à associer à I' $E C D_{2} \mathrm{O}$, nous avons finalement choisi le poids à l'injection dont nous disposions pour toutes les brebis. On peut conclure de l'étude de la relation d'estimation de I'ECM par I'ECD $2 \mathrm{O}$ que cette dernière représente plus l'eau présente au moment de l'injection ou, peut être mieux, au moment de l'équilibre que l'eau présente au moment de l'abattage. Le décalage temporel de $24 \mathrm{~h}$ entre l'injection et l'abattage entraîne des variations de poids des contenus digestifs dues notamment à la non distribution des rations le matin de l'abattage : le poids à l'abattage est donc un poids "à jeûn " ou " à contenus digestifs minimum ». Par contre, le poids à l'injection et les autres poids que nous avons testés, poids $7 \mathrm{~h}$ après l'injection ou poids moyen, sont des poids "pleins " c'est-à-dire avec des contenus digestifs importants, voire maximum. Des résultats comparables ont été trouvés par Foot, Skedd et Mc Farlane (1979) sur des brebis n'ayant pas accès à la nourriture et à l'eau entre l'injection et l'abattage $7 \mathrm{~h}$ après. Associé à l'espace de diffusion de l'eau tritiée, le poids à l'abattage relativement "à jeûn " est un moins bon estimateur des lipides que le poids à l'injection mesuré vraisemblablement après consommation d'une partie de la ration distribuée le matin. On peut donc conclure que dans nos conditions de mesure on doit utiliser avec $\mathrm{I}^{\prime} E \mathrm{CD}_{2} \mathrm{O}$ un poids mesuré après consommation d'une partie de la ration ; ceci est réalisé pour le poids à l'injection si une distribution alimentaire a lieu approximativement $2 \mathrm{~h}$ avant cette dernière comme dans la procédure proposée plus haut.

En ce qui concerne le choix du marqueur, l'ensemble de nos résultats montre que l'eau lourde est un aussi bon marqueur que l'eau tritiée. Son dosage est maintenant rapide et précis, grâce aux améliorations apportées par Tissier et al. (1978) ; l'erreur associée au dosage proprement dit est faible en regard de celles dues aux différentes procédures discutées précédemment pour la mise en œuvre de la technique. Le coût de l'eau lourde reste certes assez élevé. Elle peut cependant être utilisée sans précautions particulières et surtout sans la peur, justifiée ou non, 
de la contamination par un produit radioactif comme ce serait le cas en utilisant l'eau tritiée.

Influence du stade physiologique sur les relations entre composants de la masse corporelle. - L'introduction du stade physiologique dans les relations entre le poids vif à l'abattage, la quantité d'eau (ECM) et de lipides (ou d'énergie) corporels permet d'améliorer de manière hautement significative la précision de ces relations (relations 1 et 6 ). A même poids vif et à même quantité d'eau corporelle, les quantités de lipides présentes sont plus faibles chez les brebis en lactation que chez les brebis sèches ou gestantes.

En fait, l'eau corporelle mesurée à l'abattage est la somme de trois compartiments plus ou moins développés selon le stade physiologique : l'eau du contenu digestif, celle des organes liés à la reproduction (mamelle et utérus avec éventuellement son contenu) et celle du reste de l'organisme.

Pour l'eau du contenu digestif, le tableau 1 comme l'analyse des données individuelles, montrent bien qu'elle ne dépend pas seulement du poids de l'animal, mais aussi de son stade physiologique. Aussi pour déterminer l'effet stade sur les autres compartiments, on a calculé la relation entre la quantité de lipides (L), le poids vif vide et l'eau du corps vide. Comme dans le cas précédent (équation 6) la relation est améliorée lorsque le stade physiologique est introduit en facteur, celui-ci étant significatif ( $p<0,05$ ) dans l'équation (9).

(9) $L_{\mathrm{kg}}=0,955 \mathrm{PVV}_{\mathrm{kg}}-1,177 \mathrm{ECV}_{\mathrm{kg}}+\mathrm{S}_{\mathrm{kg}} ; \mathrm{R}^{2}=0.955$ et $\mathrm{ETR}_{\mathrm{kg}}=0,458$ où $S=-1,873$ pour les brebis sèches, $-0,938$ pour les brebis gestantes, $-2,166$ et $-2,115$ pour les brebis allaitantes en $1^{\text {re }}$ et $6^{\mathrm{e}}$ semaine de lactation.

Les différences de poids de lipides obtenues dans l'équation (6) selon le stade physiologique ne s'expliquent donc pas par les seules modifications du poids d'eau dans le tube digestif induites par ces différents stades.

Si l'on tient compte en outre de l'eau contenue dans l'utérus gravide, il est possible d'éliminer l'effet stade physiologique (NS).

(10) $L_{k g}=0,968$ (PVV-utérus) ${ }_{k g}-1,243(E C V \text { eau-utérus })_{k g}-0,705$;

$$
\mathrm{R}^{2}=0,944 \text { et } \mathrm{ETR}_{\mathrm{kg}}=0,476
$$

La présence du fœtus et des annexes est donc le facteur principal qui modifie les relations présentées ci-dessus et nécessiterait l'établissement d'équations différentes selon que les brebis sont gestantes ou non. Le nombre de femelles gestantes (7) est cependant trop réduit pour qu'une telle distinction soit possible actuellement.

Cet effet « stade physiologique ", observé dans les relations (6) et $\langle 9)$, n'est plus significatif dans l'équation (5) où PA et ECM sont remplacés par PI et $E C D_{2} \mathrm{O}$. Cette différence provient des variations de contenus digestifs et de certains compartiments liés à la reproduction. Ces variations sont à l'origne de la détérioration des relations entre $\mathrm{ECM}$ et $\mathrm{ECD}_{2} \mathrm{O}$. Une amélioration de l'estimation permettrait peut être de mettre en évidence un effet du stade physiologique. Cependant, il nous semble pour l'instant prématuré, contrairement à Cowan et al. (1979) de proposer des relations d'estimation différentes suivant le stade physiologique.

La méthode d'estimation de l'eau corporelle in vivo, par l'espace de diffusion de $\mathrm{D}_{2} \mathrm{O}$, est précise (ETR $=1,4 \mathrm{~kg}$ ) et permet donc de déterminer de manière 
satisfaisante la composition chimique des brebis. L'intérêt majeur de cette méthode est d'être non destructive, ce qui permet d'effectuer rapidement plusieurs mesures successives sur le même animal et d'éviter ainsi les erreurs d'échantillonnage qu'implique la méthode des abattages. Cette méthode est particulièrement bien adaptée aux ovins compte tenu des faibles quantités de $D_{2} O$ injectées, donc du nombre important d'animaux utilisables en expérience.

Reçu en avril 1980.

Accepté en février 1983.

ANNEXE 1

Poids et composition chimique des brebis : données individuelles $(\mathrm{kg})$

\begin{tabular}{|c|c|c|c|c|c|c|c|c|c|c|}
\hline $\begin{array}{c}N^{\circ} \\
\text { brebis }\end{array}$ & $\begin{array}{c}P V \\
\text { injection }\end{array}$ & $\begin{array}{c}\text { PV } \\
\text { abattage }\end{array}$ & ECM & $\begin{array}{c}\mathrm{ECD}_{2} \mathrm{O} \\
\mathrm{Co}^{\circ}\end{array}$ & $\begin{array}{c}\text { Eau du } \\
\text { corps vide }\end{array}$ & $\begin{array}{l}\text { Eau du } \\
\text { rumen } \\
\text { réseau } \\
\text { feuillet }\end{array}$ & Lipides & Protéines & Cendres & $\begin{array}{c}\text { Energie } \\
\text { (Mcal) }\end{array}$ \\
\hline \multicolumn{11}{|c|}{ Brebis sèches } \\
\hline $\begin{array}{r}1 \\
2 \\
3 \\
4 \\
5 \\
6 \\
7 \\
8 \\
9 \\
10 \\
11 \\
12 \\
13 \\
14 \\
15 \\
16 \\
17 \\
18 \\
19\end{array}$ & $\begin{array}{l}51,45 \\
48,28 \\
58,95 \\
68,44 \\
47,00 \\
54,00 \\
65,00 \\
66,50 \\
63,60 \\
51,20 \\
69,20 \\
50,60 \\
60,70 \\
61,60 \\
88,40 \\
67,40 \\
46,00 \\
46,20 \\
45,20\end{array}$ & $\begin{array}{l}50,05 \\
46,48 \\
57,55 \\
65,04 \\
48,00 \\
53,20 \\
64,00 \\
65,20 \\
63,00 \\
51,20 \\
68,00 \\
50,40 \\
59,30 \\
58,70 \\
84,40 \\
65,80 \\
44,00 \\
46,31 \\
45,59\end{array}$ & $\begin{array}{l}31,85 \\
28,09 \\
28,28 \\
34,75 \\
33,74 \\
32,26 \\
38,64 \\
38,78 \\
39,30 \\
33,97 \\
35,49 \\
32,22 \\
35,54 \\
41,15 \\
38,47 \\
43,18 \\
30,62 \\
29,17 \\
33,62\end{array}$ & $\begin{array}{l}33,55 \\
29,71 \\
30,06 \\
37,80 \\
32,83 \\
32,60 \\
38,18 \\
39,59 \\
41,31 \\
35,62 \\
37,17 \\
34,09 \\
37,66 \\
45,80 \\
41,70 \\
44,46 \\
31,88 \\
28,44 \\
33,82\end{array}$ & $\begin{array}{l}23,04 \\
19,54 \\
24,22 \\
29,24 \\
24,59 \\
25,82 \\
28,67 \\
27,05 \\
29,30 \\
24,66 \\
27,07 \\
22,91 \\
26,13 \\
30,58 \\
30,21 \\
29,57 \\
20,65 \\
22,20 \\
23,12\end{array}$ & $\begin{array}{l}4,86 \\
3,94 \\
3,04 \\
5,56 \\
6,19 \\
3,91 \\
6,98 \\
8,61 \\
7,84 \\
5,94 \\
5,75 \\
6,19 \\
6,35 \\
6,85 \\
5,96 \\
9,26 \\
7,38 \\
5,24 \\
8,72\end{array}$ & $\begin{array}{r}8,72 \\
9,85 \\
19,50 \\
19,51 \\
5,10 \\
11,82 \\
14,88 \\
15,85 \\
13,21 \\
7,99 \\
22,07 \\
9,48 \\
13,68 \\
6,03 \\
34,39 \\
12,08 \\
5,21 \\
7,55 \\
2,78\end{array}$ & $\begin{array}{l}5,55 \\
5,29 \\
6,55 \\
6,51 \\
5,92 \\
6,63 \\
7,68 \\
7,35 \\
7,70 \\
6,44 \\
7,59 \\
6,03 \\
7,00 \\
8,10 \\
8,48 \\
7,47 \\
5,53 \\
5,78 \\
5,80\end{array}$ & $\begin{array}{l}1,84 \\
1,55 \\
1,68 \\
2,04 \\
1,83 \\
1,61 \\
1,61 \\
2,03 \\
1,78 \\
1,78 \\
1,92 \\
1,73 \\
1,99 \\
2,23 \\
2,23 \\
1,91 \\
1,71 \\
1,95 \\
1,52\end{array}$ & $\begin{array}{r}111,31 \\
120,69 \\
219,81 \\
220,25 \\
80,01 \\
147,69 \\
183,84 \\
188,51 \\
166,34 \\
110,12 \\
249,17 \\
122,70 \\
166,75 \\
100,82 \\
368,70 \\
154,25 \\
78,50 \\
102,32 \\
57,66\end{array}$ \\
\hline \multicolumn{11}{|c|}{ Brebis gestantes } \\
\hline $\begin{array}{l}20 \\
21 \\
22 \\
23 \\
24 \\
25 \\
26\end{array}$ & $\begin{array}{l}65,87 \\
48,95 \\
65,20 \\
69,00 \\
83,50 \\
70,50 \\
79,50\end{array}$ & $\begin{array}{l}65,77 \\
47,75 \\
62,00 \\
67,37 \\
82,49 \\
68,79 \\
79,50\end{array}$ & $\begin{array}{l}37,99 \\
30,20 \\
38,06 \\
41,28 \\
52,95 \\
42,39 \\
50,14\end{array}$ & $\begin{array}{l}37,95 \\
30,15 \\
40,87 \\
46,44 \\
55,83 \\
46,16 \\
54,19\end{array}$ & $\begin{array}{l}30,70 \\
26,90 \\
31,43 \\
34,56 \\
46,50 \\
33,99 \\
41,48\end{array}$ & $\begin{array}{l}5,41 \\
1,97 \\
4,53 \\
5,00 \\
4,21 \\
5,20 \\
5,00\end{array}$ & $\begin{array}{r}16,13 \\
8,33 \\
13,72 \\
13,92 \\
14,48 \\
14,11 \\
15,27\end{array}$ & $\begin{array}{l}6,94 \\
6,17 \\
7,60 \\
8,21 \\
9,89 \\
7,67 \\
9,02\end{array}$ & $\begin{array}{l}1,76 \\
1,70 \\
1,96 \\
2,01 \\
2,29 \\
2,08 \\
2,28\end{array}$ & $\begin{array}{l}189,21 \\
111,69 \\
169,60 \\
174,62 \\
187,98 \\
173,36 \\
191,75\end{array}$ \\
\hline \multicolumn{11}{|c|}{ Brebis allaitantes à la semaine , 1} \\
\hline $\begin{array}{l}27 \\
28 \\
29 \\
30 \\
31\end{array}$ & $\begin{array}{l}76,00 \\
42,20 \\
74,30 \\
74,00 \\
44,50\end{array}$ & $\begin{array}{l}72,90 \\
43,50 \\
72,15 \\
74,91 \\
43,30\end{array}$ & $\begin{array}{l}43,00 \\
29,56 \\
41,59 \\
42,54 \\
28,57\end{array}$ & $\begin{array}{l}49,77 \\
31,65 \\
46,11 \\
42,19 \\
29,87\end{array}$ & $\begin{array}{l}30,52 \\
19,56 \\
29,04 \\
31,14 \\
19,24\end{array}$ & $\begin{array}{l}8,61 \\
7,14 \\
9,87 \\
7,73 \\
6,17\end{array}$ & $\begin{array}{r}14,84 \\
4,48 \\
17,52 \\
19,16 \\
5,09\end{array}$ & $\begin{array}{l}8,80 \\
5,03 \\
7,67 \\
7,85 \\
5,58\end{array}$ & $\begin{array}{l}2,25 \\
1,56 \\
2,06 \\
2,16 \\
1,49\end{array}$ & $\begin{array}{r}188,71 \\
68,58 \\
206,35 \\
223,55 \\
78,36\end{array}$ \\
\hline \multicolumn{11}{|c|}{ Brebis allaitantes à la semaine , 6} \\
\hline $\begin{array}{l}32 \\
33 \\
34 \\
35 \\
36 \\
37 \\
38\end{array}$ & $\begin{array}{l}61,10 \\
53,50 \\
64,50 \\
58,00 \\
74,50 \\
70,50 \\
57,70\end{array}$ & $\begin{array}{l}60,65 \\
53,50 \\
64,15 \\
58,95 \\
70,89 \\
66,80 \\
56,20\end{array}$ & $\begin{array}{l}39,25 \\
33,02 \\
42,30 \\
37,06 \\
40,25 \\
39,94 \\
40,39\end{array}$ & $\begin{array}{l}41,10 \\
36,16 \\
43,76 \\
39,00 \\
48,01 \\
42,70 \\
44,28\end{array}$ & $\begin{array}{l}24,59 \\
24,20 \\
26,09 \\
26,11 \\
29,35 \\
28,51 \\
29,24\end{array}$ & $\begin{array}{r}10,61 \\
5,73 \\
11,78 \\
7,43 \\
6,47 \\
7,41 \\
7,58\end{array}$ & $\begin{array}{r}9,52 \\
10,52 \\
10,00 \\
10,99 \\
17,96 \\
15,52 \\
4,36\end{array}$ & $\begin{array}{l}6,88 \\
5,88 \\
6,96 \\
6,37 \\
8,14 \\
7,41 \\
7,71\end{array}$ & $\begin{array}{l}1,97 \\
1,78 \\
1,97 \\
1,88 \\
2,16 \\
1,94 \\
2,03\end{array}$ & $\begin{array}{r}126,72 \\
127,82 \\
131,23 \\
136,49 \\
212,38 \\
186,88 \\
84,39\end{array}$ \\
\hline
\end{tabular}




\section{Références}

COWAN R. J., ROBINSON J. J., GREENHALGH J. F. D., MC HATTIE I., 1979. Body composition changes in lactating ewes estimated by serial slaughter and deuterum dilution. Anim. Prod., 29, 81-90.

DONNELY J. R., FREER M., 1974. Prediction of body composition in live sheep. Austr. J. agric. Res., 25, 825-834.

FARRELL D. J., REARDON T. F., 1972. Undernutrition in gazing sheep. III. Body composition and its estimation in vivo. Austr. J. agric. Res., 23, 511-517.

FOOT J. Z., GREENHALGH J. F. D., 1970. The use of deuterium oxide space to determine the amount of body fat in pregnant blackfare ewes. Br. J. Nutr., 24, 815-825.

FOOT J. Z., SKEDD E., Mc FARLANE D. N., 1979. Body composition in lactating sheep and its indirect measurement in the live animal using tritiated water. $J$. agric. Sci., Camb., 92,6981.

KEENAN M. D., Mc MANUS W. R., 1969. Changes in the body composition and efficiency of mature sheep during loss and regain of live weight. J. agric. Sci., Camb., 72, 139-147.

PANARETTO B. A., 1963. Body composition in vivo. III. The composition of living ruminants and its relation to the tritiated water space. Austr. J. agric. Res., 14, 944-952.

PANARETTO B. A., 1968. Body composition in vivo. IX. The relation of body composition to the tritiated water spaces of ewes and wethers fasted for short periods. Aust. J. agric. Res., 19, 267-272.

ROBELIN J., 1973. Estimation de la composition corporelle des animaux à partir des espaces de diffusion de l'eau marquée : revue bibliographique. Ann. Biol. anim. Bioch. Biophys., 13, 285-305.

ROBELIN J., 1977. Estimation in vivo de la composition corporelle des agneaux à partir de l'espace de diffusion de l'eau lourde. Ann. Biol. anim. Bioch. Biophys., 17, 95-105.

SEARLE T. W., 1970. Body composition in lambs and young sheep and its prediction in vivo from tritiated water space and body weight. J. agric. Sci., Camb., 74, 357-362.

SEARLE T. W., 1970. Prediction of body composition of sheep from tritiated water space and body weight in tests of published equations. J. agric. Sci., Camb., 75, 497-500.

SMITH B. S. W., SYKES A. R., 1974. The effect of route of dosing and method of estimation of tritiated water space on the determination of total body water and the prediction of body fat in sheep. J. agric. Sci., Camb., 82, 269-275.

TILL A. R., DOWNES A. M., 1972. The measurement of total body water in sheep. Austr. J. agric. Res., 13, 335-342.

TISSIER M., THÉRIEZ M., 1978. Ovins, 403-449. In Alimentation des ruminants. INRA Publications, Versailles.

TISSIER M., ROBELIN J., PURROY A., GEAY Y., 1978. Extraction et dosage automatique rapide de l'eau lourde dans les liquides biologiques. Ann. Biol. anim. Bioch. Biophys., 18, 12231228. 